\title{
Reflets
}

Revue ontaroise d'intervention sociale et communautaire

\section{Les problèmes sociaux en Ontario français}

\section{Nérée St-Amand et Gertrude Mianda}

Volume 6, numéro 2, automne 2000

Problèmes sociaux en Ontario français

URI : https://id.erudit.org/iderudit/026313ar

DOI : https://doi.org/10.7202/026313ar

Aller au sommaire du numéro

Éditeur(s)

Reflets : Revue ontaroise d'intervention sociale et communautaire

ISSN

1203-4576 (imprimé)

1712-8498 (numérique)

Découvrir la revue

Citer cet article

St-Amand, N. \& Mianda, G. (2000). Les problèmes sociaux en Ontario français. Reflets, 6(2), 10-18. https://doi.org/10.7202/026313ar

Tous droits réservés (C) Reflets : Revue ontaroise d'intervention sociale et communautaire, 2000

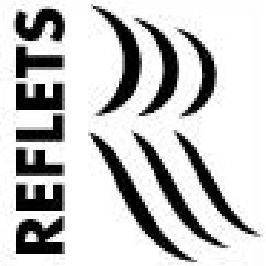

Ce document est protégé par la loi sur le droit d'auteur. L'utilisation des services d'Érudit (y compris la reproduction) est assujettie à sa politique d'utilisation que vous pouvez consulter en ligne.

https://apropos.erudit.org/fr/usagers/politique-dutilisation/ 


\section{Les problèmes sociaux en Ontario français}

Nérée St-Amand et Gertrude Mianda

On n'aide pas les pauvres en enrichissant les riches. Abraham Lincoln

Lu dans The Ottawa Citizen, le 15 octobre 2000 : sept sans-abri sont décédés au cours des dix derniers jours dans la capitale nationale. La ville en compte actuellement cinq mille, dont neuf cents enfants. Depuis 1995, aucun budget n'a été alloué au logement social. Quinze mille noms figurent actuellement sur la liste d'attente de logements subventionnés et la période d'attente est d'environ sept ans. (The Ottawa Citizen A 13). Sur la même page du quotidien, le président de la Région d'OttawaCarleton, Bob Chiarelli, affirme pouvoir compter sur un milliard de dollars pour gérer les conséquences de la croissance économique de la région. Un boom économique sans précédent! Ces dépenses seront consacrées aux infrastructures routières, aux transports en commun, etc... "L'argent n'est pas un problème ", affirme-t-il. Enfin, à la page précédente du même journal, on louange la médecine qui a sauvé des jumelles prématurées qui ont dû passer respectivement 86 jours et 144 jours à l'hôpital. Elles pesaient 1,7 livres et 1,6 livres à la naissance. Coût approximatif de leur première année de vie : un quart de million...

Lu dans The National Post, le 22 septembre 2000 : le nombre d'enfants pris en charge par la Société de l'aide à l'enfance a augmenté de $40 \%$ depuis 1996. On compte maintenant 14,200 enfants sous tutelle dans la province de l'Ontario (The National Post A8). 
Face aux problèmes sociaux de l'heure, des choix s'imposent. Multiplier les routes ou fournir du logement pour les pauvres? Une médecine de l'urgence qui coûte des millions ou une médecine préventive visant à prévenir les catastrophes? De l'argent pour les assistés sociaux ou pour les hôpitaux? Du travail obligatoire pour les assistés ou des projets à l'échelle humaine? La perpétuation de la violence ou des programmes pour la prévenir?

Les problèmes d'une société la confrontent à des choix, qu'ils soient politiques, économiques ou sociaux. Les orientations des politiques sociales en ce début de siècle, en Ontario comme ailleurs au pays, nous invitent à réfléchir à l'évolution des problèmes sociaux et des multiples facettes qu'ils recouvrent. Depuis que l'État a sonné le glas sur son rôle de Providence, il adopte un rôle plus effacé dans toutes les questions sociales : coupures dans les programmes de logement subventionné, dans les programmes d'assistance, dans les programmes de chômage et d'éducation. Dans le contexte du nouvel ordre économique qui s'impose, de nouvelles idéologies qui se confrontent, ici comme ailleurs en Occident, les politiciens privilégient des mesures moins coûteuses, les citoyens adoptent d'autres façons de penser, influencés qu'ils sont par des discours réducteurs sinon simplistes. L'heure est à la réduction des impôts et autres taxes et non à une redistribution plus juste. La mondialisation résout peut-être quelques problèmes pour certains, mais elle en crée davantage pour celles et pour ceux qui ne s'inscrivent pas dans ce mouvement. En conséquence, les pauvres restent accablés de nombreux problèmes auxquels on leur demande de faire face, avec moins de ressources. Qui est responsable? En fait, il semble plus facile de déverser les problèmes sur les individus concernés, car ainsi, nous nous déresponsabilisons des grands problèmes qui nous confrontent actuellement. Qui a tué Érick Schmitt? demande Vincent de Gaulejac (1994: 13), nous interpellant ainsi sur notre rôle de citoyens responsables.

Qu'entendons-nous au fait par problèmes sociaux? Certes, des situations difficiles, traumatisantes souvent, vécues par des individus, mais avec cette particularité que ces personnes ne sont qu'en partie responsables des situations dans lesquelles elles sont plongées. Les problèmes sociaux ont ainsi un caractère politique 
et leurs causes dépassent l'individu victime d'une situation de pauvreté, de violence, de dépendance. Analysé ainsi, un problème social nécessite évidemment une intervention structurelle plutôt qu'individuelle et isolée. Car nous savons tous qu'aucune société n'a modifié en profondeur les problèmes sociaux qui l'accablaient en ne traitant que les individus qui souffrent de malaises. L'histoire nous démontre également qu'aucune calamité qui a affecté l'humanité n'a été éliminée, ni même contrôlée en essayant de traiter les individus affectés. "Les problèmes sociaux dépendent des représentations collectives, même s'ils résultent de facteurs d'ordre divers ", nous rappelle Fernand Dumont (1994:1). Quelle représentation collective nous faisons-nous de la violence, de la pauvreté, des inégalités, du désengagement de l'État? Les articles de ce numéro tenteront, chacun à sa façon, d'apporter quelques éléments de réponse à cette question.

Dans les limites des pages qui suivent, nous ne pouvons certes pas présenter un éventail complet des problèmes sociaux en Ontario français. Nous ne faisons qu'aborder modestement quelques situations complexes, chargées d'histoire et de rebondissements, que nos dirigeants veulent souvent traiter comme des problèmes individuels. Il est certes plus facile de dire de telle personne qu'elle ne sait pas s'adapter que de s'attaquer aux causes structurelles des problèmes qui l'accablent. Pour l'État et les professionnels de l'aide, il est moins menaçant d'isoler les problèmes et les gens que de les voir devenir solidaires, créer des liens, des réseaux, des ressources. Dans les divers thèmes abordés dans la quinzaine d'articles qui suivent, nous verrons que les personnes tentent de devenir actrices de leur situation; qui plus est, les ressources locales analysent d'un oeil critique les interventions étatiques et professionnelles visant à faire face aux problèmes de l'heure.

Malgré le caractère limité de ce numéro, certaines tendances s'en dégagent. La mouvance des problèmes est définitivement un facteur à signaler. En effet, les problèmes sociaux évoluent avec le temps et avec les politiques et programmes des gouvernements et des organismes qui l'appuient. Par contre, les ressources sociocommunautaires doivent aussi s'ajuster rapidement à des situations 
changeantes, à des budgets menacés de coupures, à des ressources aussi précaires que les gens qu'elles désirent aider.

Qui plus est, les auteurs ont tous et toutes souligné la complexité des problèmes sociaux qu'ils et elles ont analysés. Que ce soit au niveau du travail obligatoire, de la formation professionnelle ou des soins à domicile, l'analyse des problèmes dénote un tricotage serré entre les politiques sociales et les problèmes individuels.

Plusieurs articles soulignent également le fait que le gouvernement ontarien se déresponsabilise aux dépens des communautés. Ajoutant l'insulte à l'opprobre, il s'enorgueillit d'avoir sabré dans les programmes sociaux et d'avoir limité l'intervention gouvernementale. Caroline Andrew souligne, à la fin de l'entrevue, que cette direction des services constitue un couteau à double tranchant pour toute personne engagée dans le social : cela oblige les communautés à se prendre en charge, mais elles doivent aussi rappeler à l'État qu'il ne doit pas se déresponsabiliser sur le dos des populations démunies. Deux luttes parallèles s'imposent donc : une pour défendre les intérêts de la population démunie et l'autre avec des dirigeants de plus en plus insensibles aux problèmes de société.

Enfin, chacun des articles propose des pistes possibles pour remédier aux problèmes sociaux de l'heure. Chacun à sa façon, les auteures et auteurs suggèrent des réponses, plus ou moins articulées, qui nous permettraient de voir autrement, de faire autrement. Nous sommes très redevables aux personnes qui ont contribué à ce numéro car elles nous permettent d'entrevoir des solutions aux problèmes complexes qu'ils et elles décrivent.

C'est justement cet ensemble de facteurs soutenus par une idéologie néolibérale que Caroline Andrew décrit dans l'entrevue. Misant sur son expérience académique et communautaire, elle sait poser la délicate question de l'engagement social dans un contexte de plus en plus contraignant. Elle utilise la question du logement, entre autres, pour démontrer les liens entre les situations locales, les politiques nationales et les pressions internationales. Intervenir dans un tel contexte, c'est à la fois répondre aux besoins des individus victimes de ce problème social, mais aussi prendre 
conscience que nos actions ont des répercussions bien au-delà des individus auxquels elles s'adressent.

Dans le premier article du Dossier, Jacinthe Michaud aborde justement cette nouvelle configuration des rapports entre les institutions gouvernementales et les groupes de base. En partant du concept d'espace intermédiaire, elle démontre comment les femmes se voient investies d'une responsabilité majeure face au travail. Elle illustre, grâce au programme de travail obligatoire en Ontario, comment les femmes sont actuellement coincées entre des politiques étatiques répressives et des groupes communautaires qui désirent s'affirmer. Les gouvernements ont besoin des groupes de base, mais à condition qu'ils ne soient pas trop conscientisés et qu'ils n'aspirent pas à devenir des partenaires à part entière. Selon Michaud, la conjoncture actuelle en Ontario met énormément de pression sur les groupes communautaires de femmes. Elles se voient dorénavant obligées de s'inscrire dans des programmes soi-disant productifs, alors que leur temps était consacré jusqu'alors à des activités créatrices. En somme, on fait fi du temps volontaire, celui qui consistait à rendre des services non rémunérés, pour obliger les femmes à s'inscrire dans des programmes de travail obligatoire. Les retombées sociales de ce programme sont remises en question alors que les gains du gouvernement sont interprétés comme des pertes pour les réseaux de femmes. En effet, le travail de réflexion sur l'oppression des femmes passe souvent par les groupes de conscientisation. Le gouvernement détourne ces énergies en instituant des programmes de travail "productif ". Pour ne pas perdre leur assistance sociale, les femmes se voient obligées de s'inscrire dans de telles filières. Ce faisant,l'État étouffe ces mouvements de revendication de la base. Dans un tel contexte, et pour reprendre l'expression de l'auteure, les nouvelles formes de citoyenneté sociale sont en mouvance et donc encore loin d'être achevées.

L'article de Cécile Coderre et de Madeleine Dubois porte justement sur des pratiques novatrices, des projets de mobilisation, de prise en charge par une communauté franco-ontarienne. En présentant certaines pratiques en émergence dans la région d'Ottawa-Carleton, elles illustrent les propos de Michaud et les 
préoccupations soulevées par Caroline Andrew au niveau de l'engagement local. Elles proposent une analyse des pratiques émergentes et des conditions grâce auxquelles des services de proximité peuvent être porteurs de sens et peuvent mobiliser les personnes pauvres. Des cuisines collectives, des coopératives de travail, des pièces de théâtre, un centre de formation et de développement économique, des magasins-partage, voilà quelques illustrations de la vitalité du local. En somme, cet article, comme celui de Claude Snow dans la rubrique suivante, nous propose déjà des pistes de solution aux problèmes sociaux actuels, des pistes qui passent par la valorisation du local. Coderre et Dubois mettent également l'accent sur la fragilité de ces ressources créées localement; les pratiques émancipatoires qui émergent des communautés du local se doivent d'être reconnues, valorisées, financées. La dimension communautaire et politique ressort clairement de ce texte qui valorise des initiatives ancrées dans la réciprocité.

Deux articles arbitrés sont signés par des étudiantes diplômées du programme de service social de l'Université d'Ottawa. Dans le premier, Marie-Hélène Gérome explore, par l'entremise d'une analyse documentaire, l'impact sur les auxiliaires familiales de la restructuration des soins de santé en Ontario. Ici comme dans l'article de Michaud, la responsabilisation accrue des femmes est décriée; les auxiliaires familiales se voient davantage obligées de porter la lourde responsabilité des soins aux personnes aînées. L'auteure illustre comment le domaine des soins à domicile, tel qu'orienté en Ontario, concourt à accentuer les inégalités reliées au sexe, à la classe sociale et à la race. C'est dans ce contexte qu'elle pose la question de l'impact de l'idéologie néolibérale qui prévaut actuellement et qui accorde peu de valeur au travail des soins à domicile. À l'heure où justement il est question de privatiser ces soins, Gérome propose des liens entre le travail «silencié » des femmes et les politiques sociales.

Geneviève Côté, pour sa part, présente une dimension souvent inexplorée de la vie des femmes: les pertes qu'elles subissent, les expériences douloureuses qu'elles traversent lorsqu'elles font face à la mort ou à d'autres tragédies, traumatismes, 
malaises. Ces divers événements difficiles, inhérents à la vie de toute femme, nécessitent un retour sur soi pour s'en sortir. C'est à travers un tel cheminement intérieur que la personne peut réaliser l'impact de la perte, vivre les émotions qui y sont associées et intégrer divers changement soit au niveau de ses attitudes, soit dans son quotidien.Version abrégée d'une analyse documentaire, cet article explore des écrits pertinents sur le processus de guérison se produisant de pair avec les pertes et le travail de deuil. Ce processus est étudié en privilégiant des principes d'analyse féministes et spirituels. Côté propose plusieurs pistes de réflexion en rapport avec les raisons et les explications attribuées aux deuils non résolus en particulier. Elle analyse diverses dimensions de la rupture et du processus de guérison à la lumière de ces écrits et propose des outils d'intervention applicables au travail de deuil. Les écrits choisis poussent la réflexion et le questionnement des idées couramment acceptées sur les étapes conventionnelles du travail de deuil et de la croissance à travers l'épreuve.

Dans un article portant sur le dilemme des étudiants-maîtres de minorités visibles nés au Canada, Donatille Mujawamariya nous rappelle que les problèmes sociaux débordent de ce que plusieurs conçoivent traditionnellement comme le domaine du social en nous faisant part des difficultés que vivent les étudiants et étudiantes des minorités visibles qui se préparent à une carrière d'enseignement en Ontario français. En partant d'entrevues semidirigées auprès d'enseignants-associés qui ont supervisé des étudiants de minorités visibles ayant grandi au Canada, elle révèle que la plupart des enseignants-associés ont tendance à considérer les futurs enseignants francophones de minorités visibles comme des étrangers qui doivent s'imprégner des valeurs de la communauté franco-ontarienne avant d'aspirer à une quelconque intégration dans la profession enseignante. Elle démontre, témoignages à l'appui, quelques-unes des résistances auxquelles font face les personnes provenant de cultures minoritaires qui, partant d'expériences culturelles diverses, désirent s'intégrer dans un milieu relativement clos.

Dans la rubrique Des pratiques à notre image, ce numéro propose deux articles de fond, un traitant d'un problème en Acadie, 
l'autre en Ontario. Celui de Claude Snow-une première, soit un article en provenance de l'Acadie-brosse d'abord un tableau des principales caractéristiques de la Péninsule acadienne sur le plan socio-économique. Par la suite, il décrit la conscientisation opérée grâce au Comité des 12 et propose, suite à leur expérience, une conception renouvelée de l'engagement social. Clairement, l'intervention individuelle passe par une analyse structurelle des problèmes sociaux. Les lecteurs pourront constater que les défis qui se présentent aux intervenants oeuvrant dans des milieux minoritaires acadiens s'apparentent beaucoup aux problèmes rencontrés en Ontario français. L'expérience du comité démontre que la mobilisation, l'engagement social et l'organisation de groupes populaires restent possibles. En Acadie comme en Ontario, un engagement au niveau local passe par une prise de conscience des enjeux des politiques actuelles et par la résistance aux politiques oppressives des dirigeants politiques et des intervenants professionnels.

Enfin, Alain Charpentier et Marie Nathalie Moreau étudient la nouvelle loi de déjuridiciarisation des jeunes en Ontario. Leur article sensibilisera nos lecteurs et lectrices aux conséquences de ces changements législatifs importants; les auteurs évaluent dans quelle mesure les changements législatifs proposés vont contribuer à améliorer ou non la situation des problèmes sociaux en Ontario. Le remplacement de la Loi sur les jeunes contrevenants par la Loi sur le système de justice pénale pour les adolescents (projet de loi C-3), représente une étape importante dans la mise en oeuvre de la stratégie fédérale en matière des services correctionnels. Pour l'Ontario français, les auteurs insistent sur le fait qu'il faudra que le gouvernement Harris démontre une volonté politique ferme et qu'il injecte des fonds importants afin d'améliorer la surveillance de l'application des services.

Voici un aperçu du numéro que nous vous présentons. Grâce à celui-ci, Reflets souhaite encourager les divers acteurs impliqués dans le domaine des problèmes sociaux à poursuivre leur réflexion sur les nombreuses modalités d'exclusion des individus et des groupes marginalisés et à renouveler leur engagement face au droit au travail, à la sécurité sociale et à un État responsable qui protège sans contrôler. Que les incertitudes 
que nous vivons actuellement soient l'occasion d'affirmer qu'il existe d'autres solutions que la charité privée et le capitalisme sauvage pour faire face aux problèmes sociaux de l'heure.

Nous vous souhaitons une bonne lecture.

\section{Références bibliographiques}

DUMONT, FERNAND; SIMON LANGLOIS et YVES MARTIN (1994). Traité des problèmes sociaux, Québec, Institut québécois de recherche sur la culture.

DE GAULEJAC,Vincent et Isabel TAABOADA LÉONETTI, (1994) La lutte des places, Paris, Épi. THE NATIONAL POST, Toronto, 22 Septembre 2000, A8.

THE OTTAWA CITIZEN, le 15 octobre 2000, A 12 et A13. 